\section{PP.88 IDENTIFICATION OF CLINICALLY IMPORTANT COMPONENTS OF MATERNAL AND NEONATAL COMPOSITE OUTCOMES TO ASSESS THE EFFECT OF TIMING OF DELIVERY IN WOMEN WITH MILD TO MODERATE PRE-ECLAMPSIA AT 34 TO 37 WEEKS}

doi:10.1136/archdischild-2013-303966.362

1,2F Fong, 1,2E Rogozinska, ${ }^{1,2} \mathrm{~J}$ Allotey, ${ }^{3} \mathrm{~S}$ Kempley, ${ }^{4} \mathrm{D}$ Shah, 1,2.5 Thangaratinam. 'Women's Health Research, Unit, Barts and the London School of Medicine and Dentistry, Queen Mary University London, London, UK; ${ }^{2}$ Centre of Primary Care and Public Health, Barts and the London School of Medicine and Dentistry, OMUL, London, UK: ${ }^{3}$ Centre for Paediatrics, Blizard Institute, Barts and the London School of Medicine, OMUL, London, UK; ${ }^{4} B$ arts and the London Children's Hospital, OMUL, London, UK; ${ }^{5}$ Multidisciplinary Evidence Synthesis Unit (MESH), OMUL, London, UK

Background Pre-eclampsia is associated with maternal and neonatal morbidity and mortality. Delivery is often expedited to minimise maternal complications at the risk of prematurity-related complications in the baby. The important outcomes to be evaluated in the management of pregnant women with pre-eclampsia between 34 to 37 weeks gestation is unclear.

Objective To identify the clinically important components of maternal and neonatal composite outcomes in the management of women with mild to moderate pre-eclampsia between 34 to 37 weeks by a Delphi survey of experts.

Methods A two-generational Delphi method was used to prioritise the maternal and neonatal outcomes that are considered to be important in the timing of delivery in women with mild to moderate pre-eclampsia.

Results The maternal outcomes questionnaire was sent to 20 participants and the neonatal outcomes questionnaire to 24 participants. $18 / 20$ participants $(90 \%)$ responded in the first round for maternal outcomes and $18 / 24$ participants (75\%) for neonatal outcomes. In the second round 16/17 eligible participants (94\%) responded for maternal outcomes and $18 / 18$ participants $(100 \%)$ for the neonatal outcomes. 21 maternal and 24 neonatal outcomes were evaluated in the first round and 17 maternal and 25 neonatal outcomes in the second round. We identified 17 and 17 components to be clinically important for development of the maternal and neonatal composite outcomes respectively. If accepted, we will be able to provide full details of the relevant outcomes.

Conclusion The composite maternal and neonatal outcomes will allow evaluation of the effect of intervention to reduce mortality and morbidity.

\section{PP.89 PRETERM PRE-LABOUR RUPTURE OF MEMBRANES (PPROM) - WHEN CLINICIANS ASPIRE TO FIND A BALANCE}

doi:10.1136/archdischild-2013-303966.363

\section{S Islam, S Hickey, S Hamilton. Calderdale Royal Hospital, HALIFAX, UK}

Introduction and objectives Preterm Pre-labour Rupture of Membranes complicates $2 \%$ of all pregnancies and is related to $40 \%$ of all preterm deliveries with significant neonatal and maternal risks. Balancing the risks between prematurity and maternal/fetal infection can pose difficult dilemmas for obstetricians. This study collected data on PPROM-delivery interval, maternal and fetal outcomes and examined the impact of inter-clinician variation in practise on clinical outcomes.

Methodology This retrospective observational study included 48 women with PPROM before 36 weeks of gestation between January 2011 and April 2012 at Calderdale Royal Hospital.
Results All patients with confirmed PPROM received erythromycin and steroid. Incidence of gestation $<34$ weeks at PPROM was $40 \%$; of delivery at $<34$ weeks was $28 \%$. Prolongation of pregnancy of $2-7$ days occurred in 52\%, 7-14 days in 2\% and $>14$ days in $13 \%$. Timing of induction of labour (IOL) varied from 34 to $36+6$ weeks although without any adverse outcomes. There were two neonatal deaths (NND) following spontaneous labour

Conclusion Prolongation of pregnancy of 48 hours or more occurred in a significant percentage of patients with conservative management. Prematurity played a crucial role in the two instances of NND. It is not clear that outcomes would have been different with different clinical management. This series shows that PPROM caries significant threat to perinatal outcome even with optimum obstetric care.

\section{PP.90 RISK FACTORS ASSOCIATED WITH INTRAUTERINE DEATHS - OUR EXPERIENCE AT DISTRICT GENERAL HOSPITAL}

doi:10.1136/archdischild-2013-303966.364

HZ Ling, O Mulki, R Hamid. Ealing Hospital NHS Trust, Southall, UK

Objective To identify the relationship between intrauterine death (IUD) and various demographics, medical and obstetrics risk factors relating to the local population in Ealing.

Methods There were 47 booked, singleton IUD cases amongst 7464 births in Ealing Hospital between April 2010 to September 2012. We retrospectively analysed the medical and obstetrics risk factors of IUDs (fetus $\geq 24 / 40$ with absent fetal heart activity inutero) in the local population.

Results IUD occurred in $0.63 \%$ of all birth. Females of Asian origin are at increased risk of IUD (45\%) compared to other ethnicities. Lifestyle risks like smoking and alcohol posed no significant increase in risk to the Ealing population, and consanguineous relationships only accounted for $8.5 \%$ of the cases. IUD was most prevalent amongst the young (20-25) primips (59.5\%) with BMI > $25(68 \%)$, mostly occurring between $37-40$ weeks (47.7\%). Majority of them were booked $\leq 12 / 40$ (63.8\%), had Dating and Anomaly scans, $(87.2 \%, 89.3 \%$ respectively), low risk on antenatal screening (59.5\%). Interestingly, previous caesarean section (6.3\%) was the most prevalent in obstetrics history. $68 \%$ had presented antenatally with reduced fetal movements, vaginal bleeding or abdominal pain. Maternal conditions like Diabetes (4.2\%), Obstetrics Cholestasis $(2.1 \%)$ and Preeclampsia (10\%) were not strongly associated with the IUD cases, indicating robust antenatal surveillance we provide to high risk women.

Conclusion While further studies aiming to modify IUD risk factors are needed, careful planning on timing of induction of labour should be carried out specifically in young, Asian primips with BMI $>25$ who present antenatally with significant episode(s).

\section{PP.91 RUPTURE UTERUS FOLLOWING PREVIOUS CAESAREAN SECTION IN THE SECOND AND EARLY THIRD TRIMESTER OF SUBSEQUENT PREGNANCY}

doi:10.1136/archdischild-2013-303966.365

S Hirsi-Farah, F Ajibade, P Street. Royal Berkshire Hospital, Reading, UK

Three cases of antenatal/pre-labour uterine rupture in women with scarred uterus.

Rupture uterus following lower segment caesarean section is rare occurrence.

Rupture uterus is linked with abnormal implantation of the placenta, and endometrial deficiency. In 10\% there's invasive placentation where placenta invades to deeper layers increasing the risks of bleeding; prematurity; SB/NND; hysterectomy and ICU admission. 